\title{
Themo-mechanical Interfacial Stress Analysis in Electronic Packaging at Different Temperature Conditions: Revisit Author's Work
}

\author{
D.Sujan, L. Vincent and Y. W. Pok \\ Faculty of Engineering and Science, Curtin University Malaysia \\ CDT 250, 98009 Miri, Sarawak, Malaysia
}

\begin{abstract}
The study of thermal mismatch induced stresses and their role in mechanical failure is one relevant topic to composite materials, photonic devices and electronic packages. Therefore, an understanding of the nature of the interfacial stresses under different temperature conditions is necessary in order to minimize or eliminate the risk of mechanical failure. An accurate estimate of thermal stresses in the interfaces plays a significant role in the design and reliability studies of microelectronic devices. In the microelectronic industry, from a practical point of view, there is a need for simple and powerful analytical models to determine interfacial stresses in layered structures. This review paper summarizes the work conducted by the authors in relation to the bi-layered assembly with different temperature conditions on the determination of interfacial thermal stresses. The authors have extended the case of uniform temperature model by earlier researchers of two layered structure to account for differential uniform temperatures, linear temperature gradient in the layers. The presence of a heat source in one layer (die) is also presented. Finally, the effect of bond material properties and geometry on interfacial stresses and bond material selection approach are also considered in a simple way.
\end{abstract}

\section{Introduction}

Thermo-mechanical stress develops at the interface of layered structures in electronic packaging during manufacturing (Curing) and operating stages. Since the electronic chips are getting smaller and smaller with increasing demand of power of the devices, a small deviation in the structure will cause functional and mechanical failure of the devices. Therefore, it is very crucial to accurately estimate the interfacial stresses in order to design the devices with reliability [1].

The existing uniform temperature model for bimaterial assembly is not really adequate to address the real life situation where the temperature levels of the two layers will be different. Again since there is heat flow in the materials, there will also be a temperature gradient in the layers. Thus, the existence of differential uniform temperatures as well as temperature gradient in the layers should be considered while determining the shearing and peeling stresses at the interface. As a result, a generalized form of the bi-material model is required to be constructed which should be able to take care of any temperature condition in the layers. The effect of heat generation on interfacial stresses due to the presence of a heat source in a layer is also needs to be investigated [17].

In this review paper, the authors have presented a summary of work in relation to the bi-layered assembly with different temperature conditions on the determination of interfacial thermal stresses. The authors have extended the case of uniform temperature model by earlier researchers of two layered structure to account for differential uniform temperatures, linear temperature gradient in the layers. The presence of a heat source in one layer (die) is also presented. Finally, the effect of bond material properties and geometry on interfacial stresses and bond material selection approach are also considered in a simple way.

\section{Bi-Layered uniform temperature model}

Fig.1 shows the full length of the model analyzed. AA represents the centre line of the model. The model length is taken as 2L. In the 2-D model, the model is considered to be of unit width in a direction perpendicular to the plane of the paper and the forces and moments are defined with respect to the unit width. 


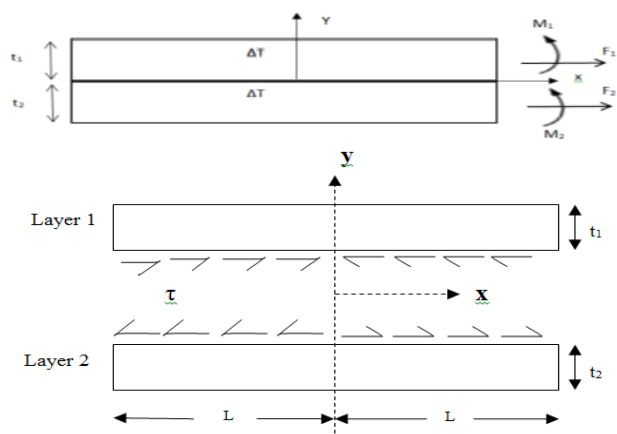

Figure 1: Full Length of the Model [1-2]

The force $\mathrm{F}$ at any section of layer is given by

$$
F=\int_{-L}^{x} \tau d x
$$

where $\tau$ is the shear stress at the interface

The compatibility condition between top and the bottom layer can be expressed in terms of displacement as:

$$
U_{x(1)}-U_{x(2)}=0
$$

where $U_{i}, \mathrm{i}=1,2$ are the axial displacements for the layers.

In our approach, we translate the above condition in a more simpler form:

$$
\epsilon_{x(1)}=\epsilon_{x(2)}
$$

where $\lambda_{\mathrm{x}(\mathrm{i})}, \mathrm{i}=1,2$ are the axial strains which is defined as $\in_{x(i)}=\frac{\partial U_{i}}{\partial x}$

By using condition (3), the model was developed by solving a second order differential equation which is much simpler compared to solution involving integrodifferential equation as in the earlier methods.

\section{The solution is based on the assumptions as follows:}

1. Thickness of the layered assembly is relatively small.

2. Each layer can be regarded as Bernoulli beam

3. Spherically bending thin plate is acted in each layer.

4. No external force acting among them.

5. Axial force due to thermal loading varies along the length and full shear length in the interface bonded layers.

6. Adhesive layer (solder bond) is very thin compared to the top and bottom layers

With reference to the Figure 1, the axial strain components at the interface of the two layers take the form,

$$
\left.\begin{array}{l}
\epsilon_{x(1)}=\alpha_{1} \Delta T+\lambda_{1} F_{1}+\frac{t_{1}}{2 R}-K_{1} \frac{\partial \tau}{\partial x} \\
\epsilon_{x(2)}=\alpha_{2} \Delta T-\lambda_{2} F_{2}-\frac{t_{2}}{2 R}+K_{2} \frac{\partial \tau}{\partial x}
\end{array}\right\},
$$

where, $K_{i}=$ interfacial shear compliances given by $K_{i}=\frac{h_{i}}{3 G_{i}} ; \mathrm{G}_{i}=$ shear modulus of rigidities given by $G_{i}=\frac{E_{i}}{2\left(1+v_{i}\right)} ; \lambda_{i}, i=$ Axial Compliances which is given by $\lambda_{i}=\frac{\left(1-v_{i}^{2}\right)}{E_{i} h_{i}}$

The shear strain components in the layers in equation (4) are expressed as follows:

(1) Strain due to shearing force $= \pm K_{i} \frac{\partial \tau}{\partial x}$

(2) Strain due to axial force $F_{i}= \pm \lambda_{i} F_{i}$;

(3) Strain due to change of curvature $= \pm \frac{h_{i}}{2 R}$;

(4) Strain due to change of temperature $=+\alpha_{i} \Delta T$ (+ve sign because $\Delta T$ is assumed to be positive and consequently the effect is an extension in the layers)

The shear stress $\tau(\mathrm{x})$ is given by,

$$
\tau=\frac{\Delta T\left(\alpha_{1}-\alpha_{2}\right)}{K \kappa \cosh (\kappa L)} \sinh (\kappa x)
$$

The peeling stress $\mathrm{P}(\mathrm{x})$ expression is given by,

$$
P=\frac{\left(t_{1} D_{2}-t_{2} D_{1}\right)}{2 D} \frac{\Delta T\left(\alpha_{1}-\alpha_{2}\right)}{K \cosh (\kappa L)} \cosh (\kappa x)
$$

In eq (5), and (6), $\kappa^{2}=\frac{\lambda}{K}$ and $K=K_{1}+K_{2}$

where $K_{1}=\frac{{ }_{1}}{3 G_{1}}$ and $K_{2}=\frac{{ }^{t_{2}}}{3 G_{2}}$ are shear stress compliances for upper and lower layer respectively.

\subsection{Bi-Material differential temperatures model}


Introducing two parameters relating to temperature and thermal expansion coefficient namely $m=\frac{\Delta T_{2}}{\Delta T_{1}}$ and $n=\frac{\alpha_{2}}{\alpha_{1}}$, equations (5) and (6) can be expressed as,

$\tau(x)=\frac{\alpha_{1} \Delta T_{1}(1-m n)}{K \kappa \cosh (\kappa L)} \sinh (\kappa x)$,

$P(x)=\frac{\left(t_{1} D_{2}-t_{2} D_{1}\right)}{2 D} \frac{\alpha_{1} \Delta T_{1}(1-m n)}{K \cosh (\kappa L)} \cosh (\kappa x)$

Where temperature changes assigned for entire Layer $1=\Delta \mathrm{T}_{1}$ and for entire Layer $2=\Delta \mathrm{T}_{2}$.

\subsection{Bi-Material Linear Temperature Gradient model}

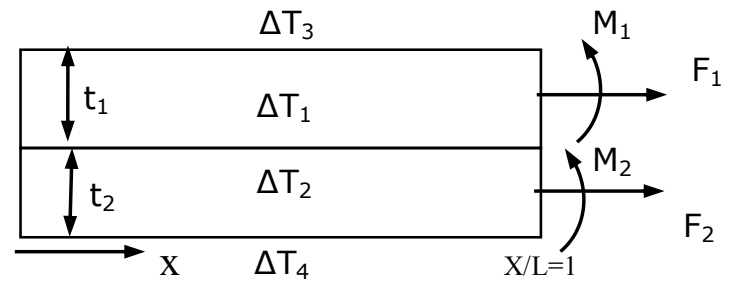

Figure 2: Bi-layered Assembly with Linear Temperature Gradients in the Layers [1].

Considering the top layer of Fig. 2, the temperature distribution throughout the thickness can be represented as shown in Fig 3.

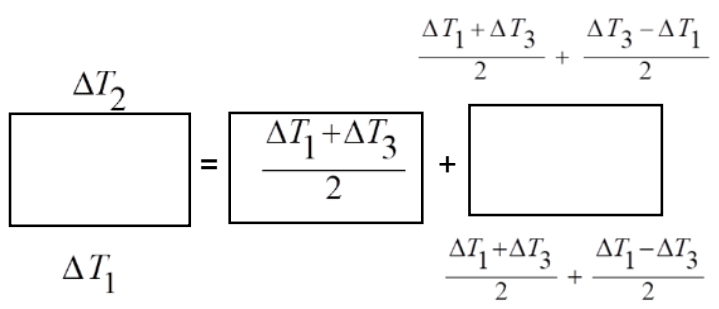

Figure 3: Linear Temperature Distribution Gradient in the Top Layer [1].

The total change of curvature of the assembly due to change of temperature is expressed by

$$
\frac{1}{R}=\frac{1}{R_{1(T)}}\left(\frac{D_{1}}{D}\right)+\frac{1}{R_{2(T)}}\left(\frac{D_{2}}{D}\right)+\frac{h F}{2 D}
$$

Now considering this modified value of $\frac{1}{R}$ in eq. (9), the eq. (7) and (8) can be reconstructed as follows:

$\tau=\frac{\alpha_{1} \Delta T_{1}\left(A_{1}+A_{2}\right)}{K \mu \cosh \mu L} \sinh \mu x$
$P=\frac{\left(h_{1} D_{2}-h_{2} D_{1}\right)}{2 D} \frac{\alpha_{1} \Delta T_{1}\left(A_{1}+A_{2}\right)}{K \cosh \mu L} \cosh \mu x$,

where $\mathrm{A}_{2}=\beta_{1} \gamma_{1}-m n \beta_{2} \gamma_{2}$,

where $\beta_{1}=\frac{\Delta T_{1}-\Delta T_{3}}{\Delta T_{1}}, \beta_{2}=\frac{\Delta T_{2}-\Delta T_{4}}{\Delta T_{2}}$,

$\gamma_{1}=\frac{t D_{1}}{2 t_{1} D}$ and $\gamma_{2}=\frac{t D_{2}}{2 t_{2} D}$.

From eq. (12) it can be observed that when gradient in materials is zero $\left(\Delta \mathrm{T}_{1}=\Delta \mathrm{T}_{3}\right)$ and $\left(\Delta \mathrm{T}_{2}=\Delta \mathrm{T}_{4}\right)$, the term $\mathrm{A}_{2}$ becomes zero and eq. (10) and (11) reduces to (7) and (8), which are the differential uniform temperature model. It is also observed that the correction factor $\mathrm{A}_{2}$ (in eq. 12) is contributed by six parameters $m, n, \beta_{1}, \beta_{2}, \gamma_{1}$, and $\gamma_{2}$.

\subsection{Derivation for heat generation in the layer (Die)}

In reality heat is generated in one of the layers say die. In this situation, the temperature distribution gradient accross the layer is expected to be quadratic ruther than linear which is expressed by the differential equation,

$\frac{d^{2} T}{d y^{2}}+\frac{G^{\prime}}{k^{\prime}}=0$

where $G^{\prime}$ and $k^{\prime}$ represent heat flux and thermal conductivity of the die material respectively [10].

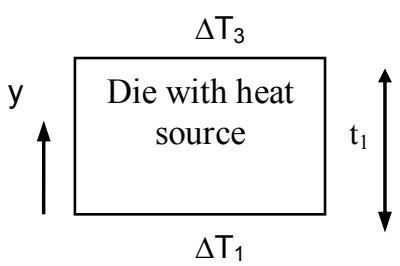

Figure 4. A Die Section with a Heat Source [1]

The expression of the $\Delta \mathrm{T}$ term can be formed by applying boundary conditions in Fig. 4 , at $y=0, \Delta \mathrm{T}=\Delta \mathrm{T}_{1}$ and at $y=t_{1}, \Delta T=\Delta T_{3}$, the solution of eq. (13) is given by,

$\Delta T=\frac{G^{\prime}}{2 k^{\prime}}\left(t_{1}-y\right) y-\frac{\left(\Delta T_{1}-\Delta T_{3}\right)}{t_{1}} y+\Delta T_{1}$,

where $\Delta T_{1}$ and $\Delta T_{3}$ represent temperature changes at the interface and top of the die respectively. Thus, so far the analytical model has taken account of the differntail temperature conditions in the layers which is more realistic from the practical packaging point of view.

\section{Analytical Model with Bond layer}


In the previous sections, a perfect bonding condition was assumed in the development of the interfacial stress model for bi-layered electronic package. However in reality, there exists a very thin layer of adhesive bonding material to attach the two layers together. There there is a need to evaluate the influence of this tiny bond layer in the analytical model. Interestingly, this surrogate bond layer may contribute significantly in eliviating the interfacial stresses by choosing appropriate bond layer parameters. In this section, the previous bi-layered perfect model in section 1 is further upgraded with the bond layer consideration. Subsequently a process flow chart is proposed to select the suitable bond using rule of mixture material for physical design and fabrication of layered assemblies.

The same analytical model which has been used in paper 1 (Title: Bond layer properties and geometry effect on interfacial thermo-mechanical stresses in bi-material electronic packaging assembly) in this conference is utilized for bond material selection, and design approach. In this paper, only the final model is presented. Figure 5 shows the free body diagram of the full length of the model.

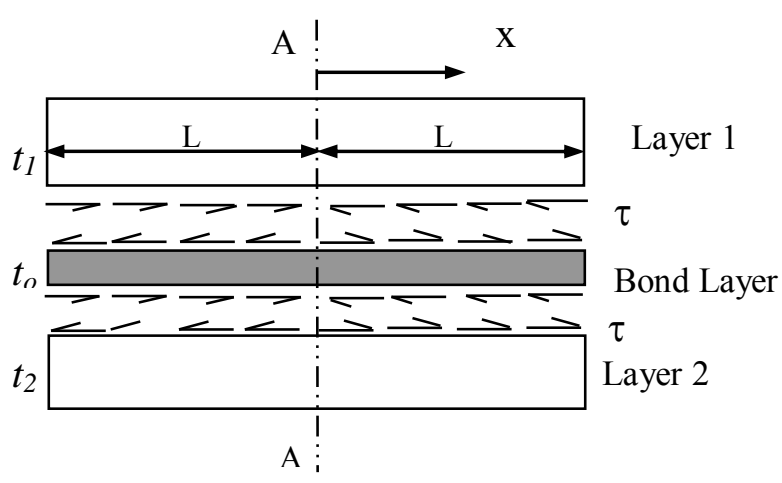

Figure 5. free-body diagram of the model $[3-4,7]$

The shear stress $\tau(\mathrm{x})$ is given by,

$$
\tau=\frac{\Delta T\left(\alpha_{1}-\alpha_{2}\right)}{K \kappa \cosh (\kappa L)} \sinh (\kappa x)
$$

The peeling stress $\mathrm{P}(\mathrm{x})$ expression is given by,

$$
P=\frac{\left(h_{1} D_{2}-h_{2} D_{1}\right)}{2 D} \frac{\Delta T\left(\alpha_{1}-\alpha_{2}\right)}{K \cosh (\kappa L)} \cosh (\kappa x)
$$

Where $t=t_{1}+t_{2}+2 t_{0}, \lambda=\lambda_{1}+\lambda_{2}+\frac{t\left(t_{1}+t_{2}\right)}{4 D}$, and $K=K_{1}+K_{2}+K_{0}$

Shear compliance for the bond layer, $K_{0}=\frac{t_{0}}{G_{0}}$

\section{The proposed bond material selection approach}

Step 1: Key in properties and geometry of chip and substrate of an arbitrary bi-layered package

Step 2: Key in range of bond layer parameters

Step 3: Key in the interfacial shearing and peeling stress

Step 4: Bond layer property and geometry parameter (for instance)

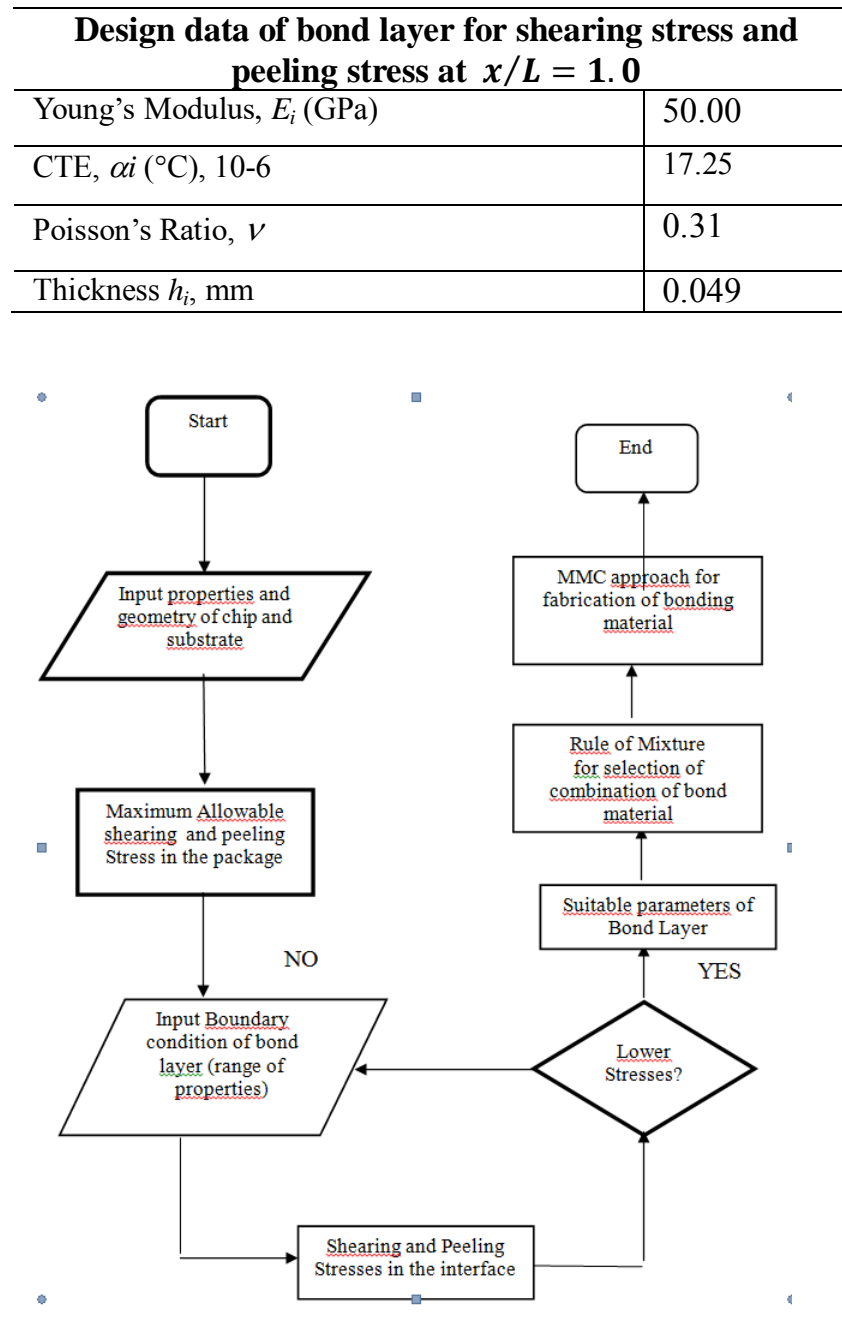

Figure 6: Rule of Mixture design for interfacial bond layer selection

Step 5: Find the volume fraction of material combination (alloy) using rule of mixture

The parametric study carried out earlier concluded that the dominant factors of bond layer in minimizing interfacial stresses in the attached layers are: elastic modulus, $E_{i}$ and thickness, $t_{i}$. Since the thickness of bond layer is a physical property that can be altered, therefore the application of rule of mixture in selecting the material combination for bond layer is focusing on elastic modulus. 
The equation for rule of mixture is, $E_{c}=f E_{A}+(1-f) E_{B}$ $f=\frac{V_{f}}{V_{f}+V_{m}}$ (volume fraction); where $E_{A}=$ Property of material A and $E_{B}=$ Property of material A

\section{Example combination 1: Tin-antimony alloy}

$$
E_{c}=f E_{A}+(1-f) E_{B}
$$

$E_{C}=$ Elastic modulus of desired bonding material, 50 $\mathrm{GPa} ; E_{A}=$ Elastic modulus of tin, $43.0 \mathrm{GPa} ; E_{B}=$ Elastic modulus of antimony, $55.0 \mathrm{GPa}$

$$
f=\frac{E_{C}-E_{B}}{E_{A}-E_{B}}=\frac{50.0-55.0}{43.0-55.0}=0.417
$$

Therefore, $41.7 \%$ of tin and $59.3 \%$ of antimony is required to manufacture tin-antimony alloy bond layer with desired Young modulus, which is $50.0 \mathrm{GPa}$ in order to minimize the interfacial stresses in the silicondiamond electronic package.

Step 6: Fabrication of MMC composite material based on the combination received from Rule of Mixture

\section{Conclusions}

This review paper summarizes the work conducted by the authors in relation to the bi-layered assembly with different temperature conditions on the determination of interfacial thermal stresses. The authors have extended the case of uniform temperature model by earlier researchers of two layered structure to account for differential uniform temperatures, linear temperature gradient in the layers. The presence of a heat source in one layer (die) is also presented. Finally, the effect of bond material properties and geometry on interfacial stresses and bond material selection approach are also considered in a simple way.

\section{Acknowledgement}

The authors thankfully acknowledge the research facilities and infrastructure provided by the Curtin University Malaysia and the financial assistance provided by the Ministry of Higher education (Malaysia) under FRGS scheme, Grant reference Code: FRGS/2/2013/SG06/CURTIN/02/1

\section{References}

1. Sujan, D., Murthy, M. V. V., Seetharamu, K. N., \& Hassan, A. Y. (2005). Complete model for interfacial stresses of a two layered structure. Paper presented at the Proceedings of the 6th International Conference on Thermal, Mechanical and Multi-Physics Simulation and Experiments in Micro-Electronics and Micro-Systems - EuroSimE 2005, , 2005 454461. doi:10.1109/ESIME.2005.1502848

2. Debnath, S., Rahman, M. E., Engida, W. D., Murthy, M. V. V., \& Seetharamu, K. N. (2011). Bi-layered model of interfacial thermal stresses with the effect of different temperatures in the layers doi:10.4028/www.scientific.net/AMM.87.63

3. Sujan, D., Woldemichael, D. E., Murthy, M. V. V., \& Seetharamu, K. N. (2011). Effect of bond layer on bimaterial assembly subjected to uniform temperature change. Journal of Electronic Packaging, Transactions of the ASME, 133(4) doi:10.1115/1.4005294

4. Sujan, D., Murthy, M. V. V., Seetharamu, K. N., \& Hassan, A. Y. (2005). Engineering model for interfacial stresses of a heated bimaterial structure with bond material used in electronic packages. Journal of Microelectronics and Electronic Packaging, 2(2), 132-141. doi:10.4071/1551-48972.2.132

5. Brown, W. D. (1998). Advanced Electronic Packaging with Emphasis on Multichip Modules. ed.

6. Suhir, E. (1989). Interfacial stresses in bimetal thermostats. Journal of Applied Mechanics, Transactions ASME, 56(3), 595-600. doi:10.1115/1.3176133

7. Sujan, D., Pang, X. B., Rahman, M. E., \& Reddy, M. M. (2014). Performance of solder bond on thermal mismatch stresses in electronic packaging assembly doi:10.4028/www.scientific.net/MSF.773-774.242 